\title{
DÜBLIN
}

Technological University Dublin ARROW@TU Dublin

2006-01-01

\section{An approach to teaching PID controller tuning}

Aidan O'Dwyer

Technological University Dublin, aidan.odwyer@tudublin.ie

Follow this and additional works at: https://arrow.tudublin.ie/engscheleart

Part of the Controls and Control Theory Commons

\section{Recommended Citation}

O'Dwyer, Aidan : An approach to teaching PID controller tuning. Proceedings of the 7th IFAC Symposium on Advances in Control Education, Madrid, Spain, 2006. doi:10.21427/y1e0-2145

This Conference Paper is brought to you for free and open access by the School of Electrical and Electronic Engineering at ARROW@TU Dublin. It has been accepted for inclusion in Conference papers by an authorized administrator of ARROW@TU Dublin. For more information, please contact arrow.admin@tudublin.ie, aisling.coyne@tudublin.ie, gerard.connolly@tudublin.ie.

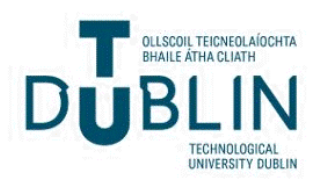




\title{
AN APPROACH TO TEACHING PID CONTROLLER TUNING
}

\author{
Aidan O'Dwyer \\ School of Control Systems and Electrical Engineering, \\ Dublin Institute of Technology, Kevin St., Dublin 8, Ireland
}

\begin{abstract}
This paper discusses an approach to the teaching of PID controller tuning methods to students in control engineering at Dublin Institute of Technology. The method involves analytically calculating the gain margin, phase margin and maximum sensitivity for PI and PID controlled systems whose process is modelled in first order lag plus time delay (FOLPD) form. Students can examine the performance of many tuning rules from graphical results, allowing insight to be developed as to the most rational choice of the tuning rule for the application. Some preliminary work done to develop an expert system to allow a greater automation of the procedure for recommending a tuning rule, for user defined requirements, is also described. Copyright (C) 2006 IFAC
\end{abstract}

Keywords: Education, PID control, processes with time delay.

\section{INTRODUCTION}

Engineering is concerned with understanding and directing the materials and forces of nature for the use and convenience of humankind. Control engineering is concerned with the understanding and control of machines, processes and industrial automation systems to provide useful economic products for society. Control engineering is based on foundations of feedback theory and linear systems analysis. It is not limited to any engineering discipline but is equally applicable to aeronautical, chemical, mechanical, environmental, civil and electrical engineering (Dorf and Bishop, 2001).

The design and implementation of proportional integral (PI) and proportional integral derivative (PID) controllers is a central part of any control engineering course, as such controllers are used in 95\% of industrial applications. The requirement to choose either two or three controller parameters has meant that the use of tuning rules (formulae) to determine these parameters is popular. The author has tabulated 443 such tuning rules in the literature to specify the PI controller terms, with 691 tuning rules defined to specify the PID controller parameters (O'Dwyer, 2006). Though the use of tuning rules is practically important, it is a challenge to allow students to gain perspective on the advantages and limitations inherent in the method. Traditionally, the topic has been taught by briefly considering the major classes of tuning rule methods, followed by a more detailed study, often in the laboratory, of one or more tuning rules (typically the process reaction curve tuning method of Ziegler and Nichols (1943) and the ultimate cycle method of Ziegler and Nichols (1942)). A number of textbooks offer a more comprehensive approach (e.g. Ogunnaike and Ray (1994), Marlin (2000), Wilkie et al. (2002), Seborg et al. (2004)), though the tuning rule methods discussed are presented in a stand-alone manner. To the authors' knowledge, no textbook gives a comprehensive approach to choosing a tuning rule based on a performance specification and knowledge of the process to be controlled. 
This paper will describe such an approach to tuning rule selection taught at the Dublin Institute of Technology to students taking control engineering as an option subject in both the final year of the fouryear honours Batchelor of Engineering (B.E.) degree in Electrical/Electronic Engineering and the one-year taught Masters of Engineering (M.E.) degree in Advanced Engineering. The gain margin, phase margin and maximum sensitivity performance criteria used to assess the tuning rules, as a function of the ratio of time delay to time constant of a first order lag plus time delay (FOLPD) model of the process, are analytically developed. This analytical development enhances student understanding of design compromises. Subsequently, the computer analysis tool MATLAB/SIMULINK is used to allow students to examine the performance of many tuning rules by generating graphical results; insight into the most rational choice of the tuning rule, for the application, is thus developed. Some work done to develop an expert system to allow greater automation of the procedure for recommending a tuning rule will be described; the experience of the author is that the expert system increases student motivation as well as providing a platform for further project work. The teaching approach is based on research work previously published by Ho et al. (1995), (1996) and O’Dwyer (1998), (2001).

The paper is organised as follows. The formulae (developed in detail with the ME students) for analytically calculating the gain margin, phase margin and maximum sensitivity, for the PI compensated process, are outlined in Section 2. Graphical results showing the performance and robustness of FOLPD processes, compensated with sample PI and PID tuning rules, are shown in Section 3. Section 4 outlines some work done on an expert system implementation of the method, for teaching purposes. Pedagogic issues are discussed in Section 5 , with conclusions of the work drawn in Section 6.

\section{THE ANALYTICAL CALCULATION OF GAIN MARGIN, PHASE MARGIN AND MAXIMUM SENSITIVITY - PI CONTROLLER}

Space considerations dictate that only an outline of the analytical calculations can be provided. The controller and process model are respectively given by

$$
\mathrm{G}_{\mathrm{c}}(\mathrm{s})=\mathrm{K}_{\mathrm{c}}\left(1+\frac{1}{\mathrm{~T}_{\mathrm{i}} \mathrm{s}}\right)
$$

and

$$
\mathrm{G}_{\mathrm{m}}(\mathrm{s})=\frac{\mathrm{K}_{\mathrm{m}} \mathrm{e}^{-\mathrm{s \tau} \mathrm{m}}}{1+\mathrm{s} \mathrm{T}_{\mathrm{m}}}
$$

The controller is a so-called ideal PI controller; 172 tuning rules have been defined for the control of processes modelled in FOLPD form using this controller (O’Dwyer, 2006). Then

$$
G_{m}(j \omega) G_{c}(j \omega)=\frac{K_{m} e^{-j \omega \tau_{m}}}{1+j \omega T_{m}} \frac{K_{c}\left(j \omega T_{i}+1\right)}{j \omega T_{i}}
$$

From the definition of gain and phase margin, the following sets of equations are obtained:

$$
\begin{aligned}
& \phi_{m}=\arg \left[G_{c}\left(j \omega_{g}\right) G_{m}\left(j \omega_{g}\right)\right]+\pi, \\
& A_{m}=\frac{1}{\left|G_{c}\left(j \omega_{p}\right) G_{m}\left(j \omega_{p}\right)\right|}
\end{aligned}
$$

where $\omega_{\mathrm{g}}$ and $\omega_{\mathrm{p}}$ are given by

$$
\begin{gathered}
\left|G_{c}\left(j \omega_{g}\right) G_{m}\left(j \omega_{g}\right)\right|=1 \text { and } \\
\arg \left[G_{c}\left(j \omega_{p}\right) G_{m}\left(j \omega_{p}\right)\right]=-\pi
\end{gathered}
$$

Analytical formulae may be directly calculated for $\omega_{\mathrm{g}}$ and $\omega_{\mathrm{p}}$, and subsequently for the gain and phase margin of the compensated system, for each of the tuning rules, as a function of $\tau_{\mathrm{m}} / \mathrm{T}_{\mathrm{m}}$ (O'Dwyer, 2006). The maximum sensitivity (the reciprocal of the shortest distance from the Nyquist curve to the $(-1,0)$ point on the Rl-Im axis) may also be directly determined.

The method can be extended to the determination of the gain margin, phase margin and maximum sensitivity of FOLPD process models, compensated by the classical PID controller structure, and the ideal PID controller structure (with some approximation), in a straightforward manner. These controller structures are given by

$$
\mathrm{G}_{\mathrm{c}}(\mathrm{s})=\mathrm{K}_{\mathrm{c}}\left(1+\frac{1}{\mathrm{~T}_{\mathrm{i}} \mathrm{s}}\right)\left(\frac{1+\mathrm{sT}_{\mathrm{d}}}{1+\mathrm{s} \alpha \mathrm{T}_{\mathrm{d}}}\right)
$$

and

$$
\mathrm{G}_{\mathrm{c}}(\mathrm{s})=\mathrm{K}_{\mathrm{c}}\left(1+\frac{1}{\mathrm{~T}_{\mathrm{i}} \mathrm{s}}+\mathrm{T}_{\mathrm{d}} \mathrm{s}\right),
$$

respectively. 154 tuning rules have been defined for the control of processes, modelled in FOLPD form, using these controllers (O'Dwyer, 2006).

\section{SIMULATION RESULTS}

Space considerations dictate that only representative simulation results may be provided; an extensive set of simulation results covering many of the PI controller and PID controller tuning rules are available (O'Dwyer, 2000). The MATLAB package has been used in the simulations. Figures 1 to 6 show how gain margin, phase margin and maximum sensitivity vary as the ratio of time delay to time constant varies, if some PI tuning rules are used (Figures 1 to 3 ) and corresponding PID tuning rules 
for the classical controller structure (with $\alpha=0.1$ ) are used (Figures 4 to 6). Figures 7 and 8 show one example of gain and phase margin comparisons between corresponding PI and PID controller tuning rules.

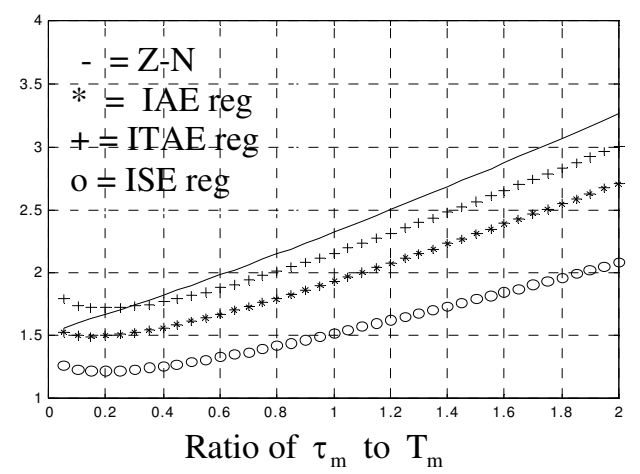

Fig. 1. Gain margin

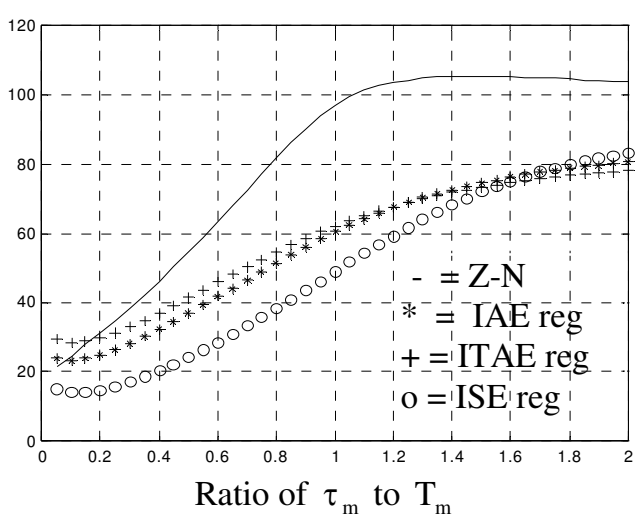

Fig. 2. Phase margin

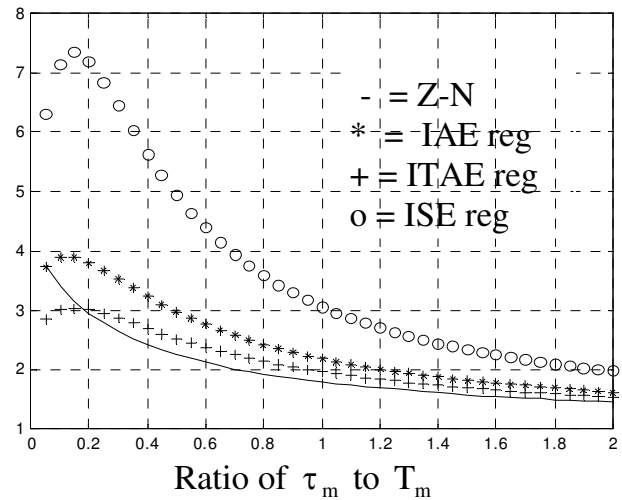

Fig. 3. Maximum sensitivity

In these results, $\mathrm{Z}-\mathrm{N}$ refers to the process reaction curve method of Ziegler and Nichols (1942); W-W refers to the process reaction curve method of Witt and Waggoner (1990); IAE reg, ISE reg and ITAE reg refer to the tuning rules for regulator applications that minimise the integral of absolute error criterion, the integral of squared error criterion and the integral of time multiplied by absolute error criterion, respectively, as defined by Murrill (1967) for PI tuning rules and Kaya and Scheib (1988) for PID tuning rules based on the classical controller structure.

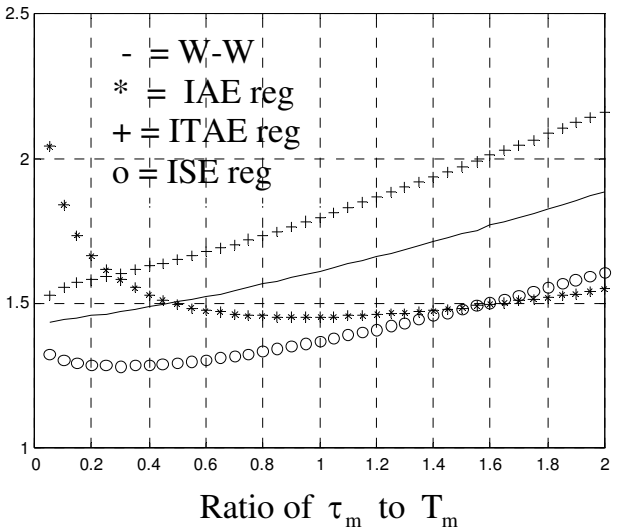

Fig. 4. Gain margin

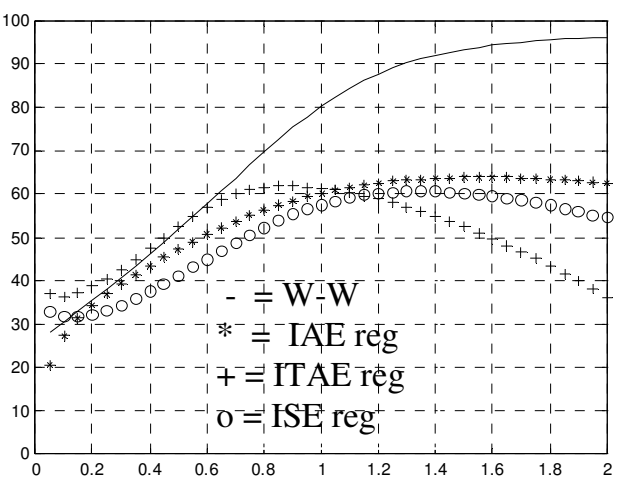

Ratio of $\tau_{\mathrm{m}}$ to $\mathrm{T}_{\mathrm{m}}$

Fig. 5. Phase margin

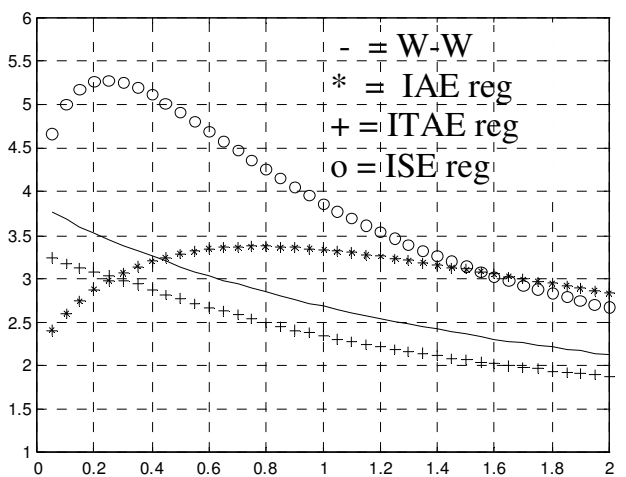

Ratio of $\tau_{\mathrm{m}}$ to $\mathrm{T}_{\mathrm{m}}$

Fig. 6. Maximum sensitivity

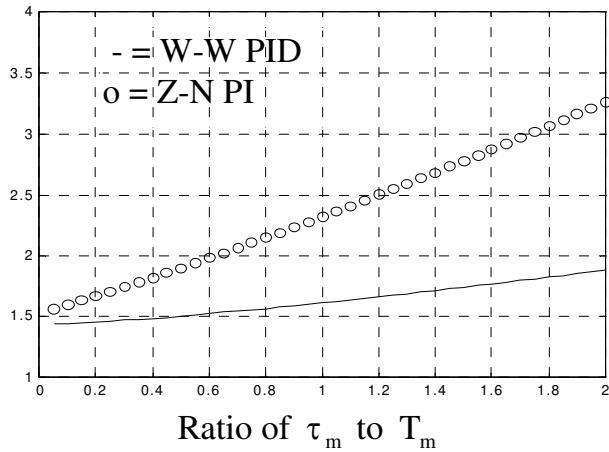

Fig. 7. Gain margin comparison 


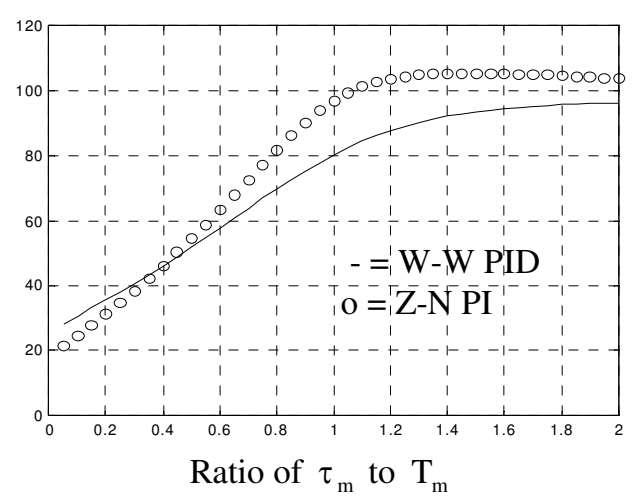

Fig. 8. Phase margin comparison

These results would allow the following general points to be outlined in the classroom:

- It is clear that the gain margin is generally less when the PID rather than the PI tuning rules are considered, over the ratios of time delay to time constant taken; the difference between the phase margins is less clear-cut. This suggests that these PID tuning rules should provide a greater degree of performance than the corresponding PI tuning rules, but may be less robust.

- Comparing the individual tuning rules, it is striking that the ISE based tuning rules have generally the smallest gain margin and also have a small phase margin, suggesting that this is a less robust tuning strategy. The results in Figures 3 and 6 confirm these comments.

- No general conclusion can be reached as to the best tuning rule (as expected); it is interesting, though, that many tuning rules may be applied at ratios of time delay to time constant greater than that normally recommended. One example may be seen in Figures 4 to 6, where the gain margin, phase margin and maximum sensitivity (associated with the use of the PID tuning rule for obtaining minimum IAE in the regulator mode) tends to level out when the ratio of time delay to time constant is greater than 1; normally, the tuning rule is used when the ratio is less than 1 (Murrill, 1967). On the other hand, it is clear from Figures 7 and 8 that there is a significant degradation of performance when using the PID tuning rule of Witt and Waggoner (1990) and the PI tuning rule of Ziegler and Nichols (1942) for large ratios of time delay to time constant, which is compatible with application experience.

- The decision between the use of a PI and PID controller to compensate the process, depends on the ratio of time delay to time constant in the FOLPD model, together with the desired tradeoff between performance and robustness, as expected. It turns out, however, that the analytical method explored allows the calculation of a far wider range of gain and phase margins for PI controllers; it is also true that stability tends to be assured when a PI controller is used (O'Dwyer, 2000). Thus, a cautious design approach is to use a PI controller, particularly at larger ratios of time delay to time constant.

\section{EXPERT SYSTEM IMPLEMENTATION}

In Section 3, data has been defined as MATLAB variables representing gain margin and phase margin values, as the ratio of time delay to time constant varies, for many of the $172 \mathrm{PI}$ controller-tuning rules for FOLPD process models (O'Dwyer, 2000). Though the graphical results based on this data gives students a good insight into the usefulness of the tuning rules (at larger time delays, for example), the sheer volume of results generated means that a more automatic means of comparing the tuning rules would be useful. Thus, some preliminary work on the development of an expert system was carried out. The expert system asks the user to input the desired range of gain and phase margin of the compensated system. The expert system will then indicate the PI tuning rules that fit the criteria, outputting the gain margin and phase margin associated with each rule, together with a recommendation.

The gain margin and phase margin data, associated with each tuning rule, was first exported to a Microsoft Access database file. A Microsoft Visual Basic (VB) front end was developed using intrinsic VB controls, to provide the user with a friendly and intuitive interface. On correct completion of a logon process, the main user screen, shown in Figure 9, is loaded and a connection to the Microsoft Access database is made using a VB data control object. The database is local to the VB programme (i.e. it is on the same PC as the VB application), though it could be put on a Local Area Network (LAN) or a Wide Area Network (WAN). The upper portion of this screen is used as an input interface. It consists of a set of text boxes and labels into which the user may enter the ratio of delay to time constant (TD/TC on Figure 9), the required gain and phase margins and an acceptable variation (called tolerance on Figure 9) on the gain and phase margins.

The process of retrieval starts when the Execute button is clicked. A Structured Query Language (SQL) query, using parameters from the input text boxes, was used to return a record set of matching tuning rules. A VB DBGrid object, bound to the data control object, was used to display the record set. If the system finds fewer tuning rules than a low threshold value, or more tuning rules than a high threshold value, then the user is prompted to widen or narrow the default gain and phase margin variation of $10 \%$, respectively. A secondary SQL query using the tuning rule number returned by the first query, was used to access another database containing the tuning rule sources and the formulae associated with each tuning rule. The right hand panels in the lower half of the user screen (Figure 9) were used to display this information. 


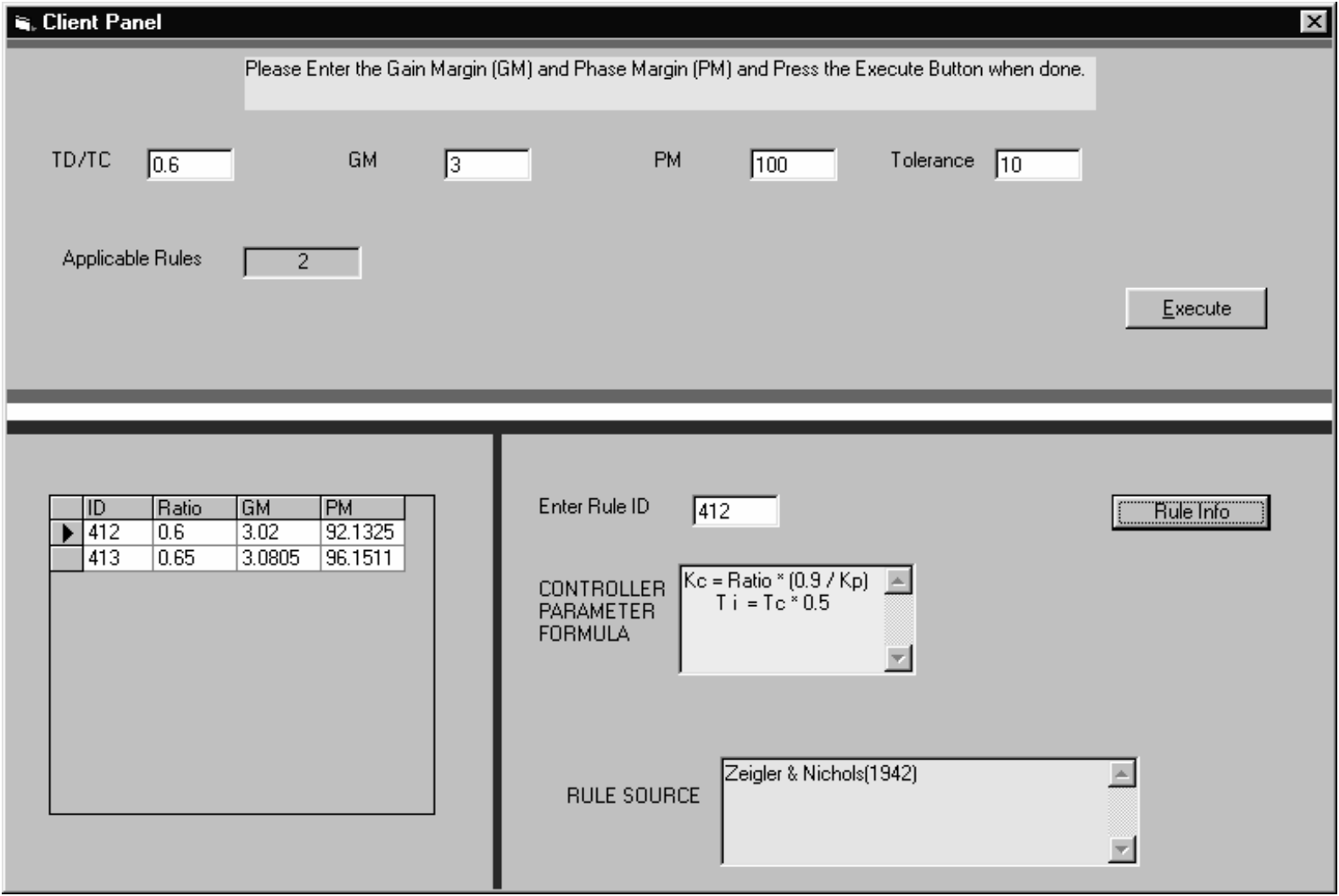

Fig. 9: Main User Screen

\section{PEDAGOGIC ISSUES}

A control engineering educator has the challenge of communicating a wide variety of concepts, ideas and techniques, to provide students of the discipline with both a strong theoretical base and good practical ability. In addition, the educator increasingly has the responsibility of providing students with the fundamental skills that are required for life long self-learning. Theoretical issues, which often involve mathematical and physical analysis, have tended to be taught in the classroom. Practical ability, which requires intuition and insight, has been traditionally conveyed through extensive laboratory work. However, time available for classroom and laboratory work has been reduced, in response to pressure to reduce class contact hours and the increasing desire to facilitate student self-learning. The use of computer aided design and analysis tools, which facilitate student self-learning and enhance theoretical understanding and practical ability, is one way to increase learning efficiency. Computer simulation also allows the study of a greater range of problems than would be possible through either mathematical analysis or laboratory work.

The experience of the author is that the use of the teaching method outlined allows a greater integration between theory and practice than heretofore. As mentioned, the theoretical aspects outlined in Section 2 are explored in detail with the ME students, and in outline with the BE students; the authors experience is that BE students, on balance, derive more benefit from a greater emphasis on computer simulation. The author has not attempted to formally measure the improvement in learning experienced. However, less laboratory time is required compared to a more traditional approach to teaching the topic, an important issue when formal laboratory time in the subject is less than $50 \%$ of what it was five years ago. On the other hand, it is the authors' experience that (perhaps, surprisingly) some engineering students are alienated by computer simulation tools and would benefit from more traditional, hands-on laboratory work. The authors' development work on the expert system is partly motivated by this concern, as well as the wish to improve learning efficiency further. The expert system allows the learner to quickly obtain a candidate tuning rule, based on user data input; the learner can then implement and test this tuning rule in the laboratory, in a hands-on environment. More motivated students do use the capabilities of the expert system and the MATLAB simulation results to increase their understanding; interestingly, proportionally more part-time students do this, perhaps because their maturity allows them to see that a little time invested by them in this activity has a disproportionate educational benefit.

Further enhancements to the expert system are presently taking place, with the aim of providing a more comprehensive learning experience by using 
recent developments in graphical user interface technology.

\section{CONCLUSIONS}

The paper has reported on the teaching of PI and PID controller tuning methods by determining the performance and robustness of a PI and PID controlled FOLPD process, with the parameters of the controllers determined by a variety of tuning rules. The method allows an analytical approach to be taken to the evaluation for a large number of tuning rules. The development of the analytical procedure increases student understanding of the concepts of gain margin, phase margin and maximum sensitivity; in addition, the plotted results allow the students to understand immediately, for each tuning rule, the performance and robustness of the compensated system, the appropriateness of the tuning rule for a given process and the range of time delay to time constant ratios over which it is sensible to apply the tuning rule. Limited comparisons of the tuning rules are possible, as is shown in Section 3. The expert system reported facilitates student motivation as well as providing a platform for further project work. In short, the approach outlined clarifies the topic of tuning rule selection and informs subsequent laboratory work. Finally, the method complements iterative methods of controller design, using Bode plots, based on gain and phase margin specifications that are also covered in the courses.

\section{REFERENCES}

Dorf, R.C. and R.H. Bishop (2001). Modern Control Systems ( $9^{\text {th }}$ Edition). Prentice-Hall International.

Ho, W.K., C.C. Hang and J.H. Zhou (1995). Performance and gain and phase margins of wellknown PI tuning formulas. IEEE Transactions on Control Systems Technology, 3, 245-248.

Ho, W.K., O.P. Gan, E.B. Tay and E.L. Ang (1996). Performance and gain and phase margins of well-known PID tuning formulas. IEEE Transactions on Control Systems Technology, $\mathbf{4}$, 473-477.

Kaya, A. and T.J. Scheib (1988). Tuning of PID controls of different structures. Control Engineering, July, 62-65.

Marlin, T.E. (2000). Process Control: designing processes and control systems for dynamic performance. Mc-Graw-Hill, $2^{\text {nd }}$ edition.

Murrill, P.W. (1967). Automatic control of processes. International Textbook Co.

O'Dwyer, A. (1998). Performance and robustness issues in the compensation of FOLPD processes with PI and PID controllers. Proc. Irish Signals and Systems Conference, Dublin Institute of Technology, Ireland, 227-234.

O'Dwyer, A. (2000). Technical Report AOD-OO12, Dublin Institute of Technology, Ireland.

O'Dwyer, A. (2001). PI and PID controller tuning rule design for processes with delay, to achieve constant gain and phase margins for all values of delay. Proc. Irish Signals and Systems Conference, National University of Ireland, Maynooth, Ireland, 96-100.

O'Dwyer, A. (2006). Handbook of PI and PID controller tuning rules ( $2^{\text {nd }}$ Edition). Imperial College Press.

Ogunnaike, B. and W. Ray (1994). Process dynamics, modeling and control. Oxford University Press.

Seborg, D.E., T.F. Edgar and D.A. Mellichamp (2004). Process dynamics and control, $2^{\text {nd }}$ edition, Wiley.

Wilkie, J., M. Johnson and R. Katebi (2002). Control engineering: an introductory course. Palgrave Ltd.

Witt, S.D. and R.C. Waggoner (1990). Tuning parameters for non-PID three-mode controllers. Hydrocarbon Processing, June, 74-78.

Ziegler, J.G. and Nichols, N.B. (1942). Optimum settings for automatic controllers. Transactions of the ASME, 64, 759-768.

Ziegler, J.G. and Nichols, N.B. (1943). Process lags in automatic control circuits. Transactions of the ASME, July, 433-444. 